\title{
Low Flow-Mediated Constriction: Prevalence, Impact, and Physiological Determinant
}

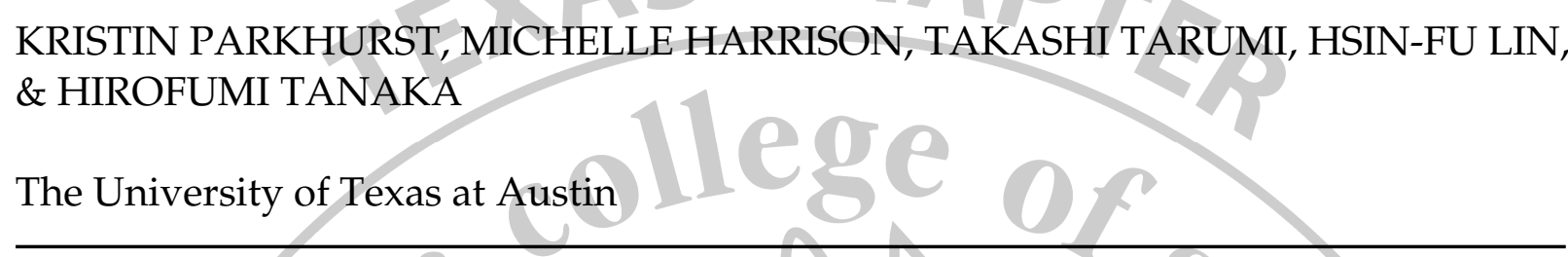

ABSTRACT

Flow-mediated dilation (FMD) is a surrogate marker for endothelial function. In the FMD procedure, arterial response during cuff inflation is not taken into consideration yet studies have demonstrated vasoconstriction, vasodilation, and no change in the brachial artery during cuff inflation. The term low flow-mediated constriction (L-FMC) has been introduced to describe the vasoconstriction that occurs in some individuals during inflation of the cuff. PURPOSE: To determine 1) if brachial artery response during cuff inflation differed in a population with varied coronary artery disease (CAD) risk factor profiles, 2) the impact of this response on the subsequent calculation of FMD, and 3) the role of arterial stiffness in this variable response. METHODS: Low flow-mediated constriction (L-FMC), "traditional" FMD, and "modified" FMD that accounts for L-FMC by using inflation diameter in place of baseline diameter in calculating FMD, were studied in a total of 46 subjects. Subjects varied in age (38-62 years) and risk factor profiles for coronary artery disease. RESULTS: During cuff inflation, brachial artery responses varied widely from $-5.6 \%$ (vasoconstriction) to $5.0 \%$ (vasodilation). When subjects were divided into healthy versus multiple risk factors $(n=34)$, L-FMC and FMD were not different between the groups but modified FMD was significantly different $(p=0.02)$. L-FMC was modestly but significantly associated with FMD ( $\mathrm{r}=0.41)$ and positively correlated with brachial artery pulse wave velocity $(\mathrm{r}=0.30)$. CONCLUSION: Our results indicate that brachial artery responses to inflation of the cuff are very variable and are associated with arterial stiffness and that accounting for so-called L-FMC may provide a more comprehensive assessment of endothelial vasodilatory function.

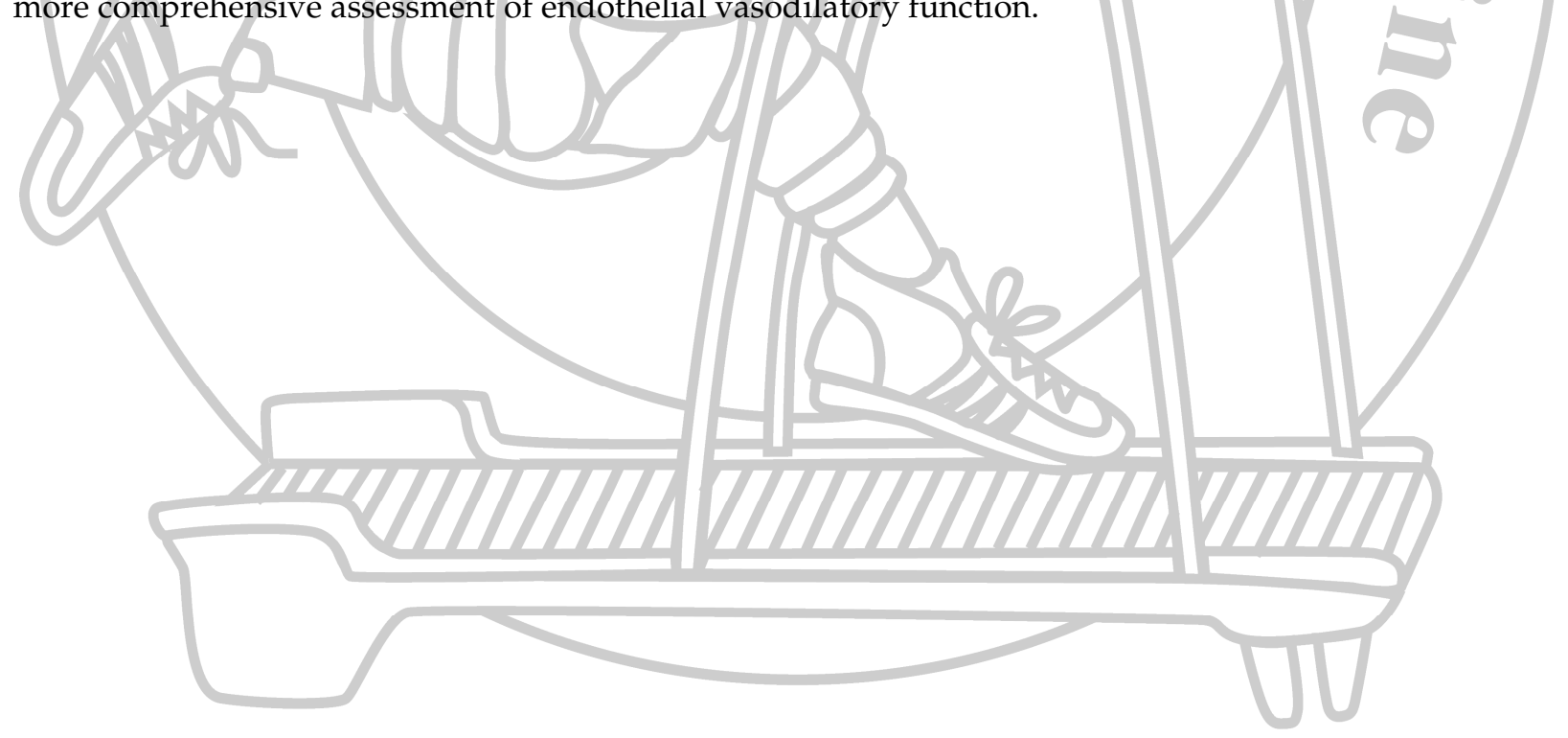

